(n)

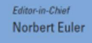
Journal of Nonlinear Mathematical Physics

\title{
Primary Branch Solutions of First Order Autonomous Scalar Partial Differential Equations via Lie Symmetry Approach
}

S. Y. Lou, Ruo Xia Yao

To cite this article: S. Y. Lou, Ruo Xia Yao (2017) Primary Branch Solutions of First Order Autonomous Scalar Partial Differential Equations via Lie Symmetry Approach, Journal of Nonlinear Mathematical Physics 24:3, 379-392, DOI:

https://doi.org/10.1080/14029251.2017.1341700

To link to this article: https://doi.org/10.1080/14029251.2017.1341700

Published online: 04 January 2021 


\title{
Primary Branch Solutions of First Order Autonomous Scalar Partial Differential Equations via Lie Symmetry Approach
}

\author{
S. Y. Lou* \\ Ningbo Collabrative Innovation Center of Nonlinear Harzard System of Ocean and Atmosphere and Faculty of Science, \\ Ningbo University, Ningbo, 315211, China, Shanghai Key Laboratory of Trustworthy Computing, East China Normal \\ University, Shanghai 200062, China \\ Ruo Xia Yao \\ School of Computer Science, Shaanxi Normal University, Xi'an, 710062, China
}

Received 12 February 2017

Accepted 20 April 2017

\begin{abstract}
A primary branch solution (PBS) is defined as a solution with $m$ independent $n-1$ dimensional arbitrary functions for an $m$ order $n$ dimensional partial differential equation (PDE). PBSs of arbitrary first order scalar PDEs can be determined by using Lie symmetry group approach companying with the introduction of auxiliary fields. Because of the intrusion of the arbitrary function, the PBSs have abundant and complicated structure. Usually, PBSs are implicit solutions. In some special cases, explicit solutions such as the instanton (rogue wave like) solutions may be obtained by suitably fixing the arbitrary function of the PBS.
\end{abstract}

Keywords: Primary branch solutions; symmetry approach; Arbitrary first order scalar PDE.

2000 Mathematics Subject Classification: 22E46, 53C35, 57S20

\section{Introduction}

It is well known that the symmetry group theory is very useful while studying exact solutions of nonlinear systems. In fact, group theory was first established by Abel and Galois to prove the nonexistence of general algebraic solution for a five degree univariate polynomial equation. Sophus Lie and Felix Klein introduced Lie symmetry algebras to study partial differential equations(PDEs) [8]. In this paper, we try to find primary branch solutions (PBSs) of arbitrary first order autonomous one component partial differential equations in any dimensions.

Definition 1.1. For an $m$ order $n$ dimensional PDE, a solution is called a PBS if it contains $m$ independent $n-1$ dimensional arbitrary functions. If only $m_{1}<m$ independent $n-1$ dimensional arbitrary functions are included in a solution, then it is called a secondary branch solution (SBS). If a solution contains some lower dimensional $\left(n_{1}<n-1\right.$ dimensional) arbitrary functions, then it is called a degenerate solution.

In fact, PBSs exist for all Painlevé integrable systems. The singularity analysis (Painlevé analysis) shows that if a system of PDEs is Painlevé integrable, then there exists a primary branch such

*Email: lousenyue@ nbu.edu.cn 
that enough arbitrary functions (same as that in the definition of the PBSs) are included in a formal series solution with respect to an arbitrary singularity manifold $[1,5,10,13]$. Thus, if one tries to find the PBSs of a PDE system by means of symmetry theory, then enough symmetries with enough arbitrary functions need to be found. Conversely, if we can find enough symmetries with enough arbitrary functions, then, we may successfully find the PBSs of the related PDE system. To make this idea clear, we study the symmetries and exact solutions of the following arbitrary $(n+1)$-dimensional first order PDE,

$$
F\left(u, u_{t}, u_{x_{1}}, \ldots, u_{x_{n}}\right)=0,
$$

where $F$ is an arbitrary function and $n$ is an arbitrary positive integer.

Obviously, there exists only one $n$ dimensional arbitrary function for the PBSs of the first order PDE (1.1). Thus, the SBSs of (1.1) are also degenerate solutions. In this paper we do not discuss degenerate solutions of (1.1). Usually, degenerate solutions of (1.1) can be recasted to PBSs for some suitable lower dimensional models.

It is worth to emphasize that various special cases of (1.1) can be widely found in many physical fields. For instance, the Hopf $(b=0)[3]$ and damped Hopf $(b \neq 0)$ [14] equations (also named Burgers and damped Burgers equations)

$$
u_{t}=a u u_{x}+b u,
$$

with arbitrary constants $a$ and $b$ is a standard model to describe shock waves [12] with and without damping.

A general Hopf equation, a model equation of gas dynamics,

$$
u_{t}=f(u) u_{x}, \quad(f(u) \text { being an arbitrary function of } u),
$$

is also widely used in hydrodynamics, multiphase flows, wave theory, acoustics, chemical engineering and other applications [7,9].

The most general two dimensional form of (1.1) with the form

$$
F\left(u, u_{x}, u_{y}\right)=0
$$

defines cylindrical surfaces whose elements are parallel to the $\{x y\}$ plane [4].

A simple multiple dimensional significant model,

$$
\sum_{i=0}^{n} a_{i} u_{x_{i}}^{2}=c, \quad c, a_{i},(i=0,1, \ldots, n), \text { being arbitrary constants }
$$

is encountered in differential games [11].

In Section 2, the symmetries and exact solutions of the general 1+1 dimensional first order autonomous scalar systems are studied by using Lie point symmetry theory. Using the same idea as in Section 2, the symmetries and exact PBSs of the first order arbitrary autonomous scalar system in any dimensions are investigated in Section 3. The last section is a short summary and discussion.

\section{Symmetries and solutions of $(1+1)$-dimensional systems}

Prior to study the general first order PDE (1.1), we investigate the symmetries and exact solutions of Eq. (1.1) in (1+1)-dimensional case. Under this case, one of primary non-degenerate branches of 
Eq. (1.1) may have the form

$$
u_{t}=F\left(u, u_{x}\right) u_{x},
$$

where the subscripts are partial derivatives and one $u_{x}$ factor is separated from arbitrary function $F\left(u, u_{x}\right)$ for simplicity later.

A symmetry, denoted by $\sigma$, of Eq. (2.1) is defined as a solution of its linearized equation,

$$
\frac{d \sigma}{d t}-F_{u} u_{x} \sigma-\left(u_{x} F\right)_{u_{x}} \frac{d \sigma}{d x}=0,
$$

which means that Eq. (2.1) is invariant under the transformation

$$
u \rightarrow u+\varepsilon \sigma
$$

with infinitesimal parameter $\varepsilon$, where

$$
\sigma=\sigma\left(x, t, u, u_{x^{i}}, i=1, \ldots, N\right)
$$

for general local symmetries ( $N$ being an arbitrary integer), and $\frac{d}{d t}$ and $\frac{d}{d x}$ are total derivatives with respect to $t$ and $x$, respectively.

However, for Lie point symmetries, (2.4) has the special form

$$
\sigma=U(x, t, u)-X(x, t, u) u_{x}-T(x, t, y) u_{t} .
$$

For any fixed $F$ in (2.1), one may find the concrete forms of (2.5) by using standard Lie symmetry group approach.

However, for arbitrary $F$, it is not difficult to find that the only Lie point symmetries are those of related to space and time translations, $u_{x}$ and $u_{t}$, in other words, for arbitrary $F$, the general solution of (2.2) with (2.5) possesses the form

$$
\sigma=x_{0} u_{x}+t_{0} u_{t}
$$

where $x_{0}$ and $t_{0}$ are arbitrary constants.

To look for more symmetries via Lie symmetry approach we can introduce two auxiliary equations

$$
\begin{aligned}
& u_{x}=u_{1}, \\
& u_{t}=u_{0} .
\end{aligned}
$$

With the help of the auxiliary system (2.7), we can look for the Lie point symmetries in the form

$$
\begin{aligned}
\sigma & =U\left(x, t, u, u_{0}, u_{1}\right)-X\left(x, t, u, u_{0}, u_{1}\right) u_{x}-T\left(x, t, u, u_{0}, u_{1}\right) u_{t}, \\
\sigma_{0} & =U_{0}\left(x, t, u, u_{0}, u_{1}\right)-X\left(x, t, u, u_{0}, u_{1}\right) u_{0 x}-T\left(x, t, u, u_{0}, u_{1}\right) u_{0 t}, \\
\sigma_{1} & =U_{1}\left(x, t, u, u_{0}, u_{1}\right)-X\left(x, t, u, u_{0}, u_{1}\right) u_{1 x}-T\left(x, t, u, u_{0}, u_{1}\right) u_{1 t}
\end{aligned}
$$

for the equation system (2.1) and (2.7), where $\sigma_{0}$ and $\sigma_{1}$ should be solutions of

$$
\begin{aligned}
& \sigma_{x}=\sigma_{1}, \\
& \sigma_{t}=\sigma_{0} .
\end{aligned}
$$


Because of the auxiliary system (2.7), the model equation (2.1) can be rewritten as

$$
u_{0}=F\left(u, u_{1}\right), \quad \text { or } \quad u=F^{\prime}\left(u_{0}, u_{1}\right), \quad \text { or } \quad u_{1}=F^{\prime \prime}\left(u, u_{0}\right),
$$

and the Lie point symmetry (2.8) can be rewritten in three different but equivalent forms by eliminating one of $u, u_{0}$ and $u_{1}$, respectively,

$$
\begin{aligned}
\sigma & =\left.\left[U\left(x, t, u, u_{0}, u_{1}\right)-X\left(x, t, u, u_{0}, u_{1}\right) u_{1}-T\left(x, t, u, u_{0}, u_{1}\right) u_{0}\right]\right|_{u=F^{\prime}\left(u_{0}, u_{1}\right)} \\
& \equiv G\left(x, t, u_{0}, u_{1}\right) \\
& =G\left(x, t, F\left(u, u_{1}\right) u_{1}, u_{1}\right) \equiv H\left(x, t, u, u_{1}\right) u_{1} \\
& =G\left(x, t, u_{0}, F^{\prime \prime}\left(u, u_{0}\right)\right) \equiv J\left(x, t, u, u_{0}\right) u_{0}
\end{aligned}
$$

Correspondingly, $\sigma_{0}$ and $\sigma_{1}$ possess also three equivalent forms. For instance, using the formula (2.11), the corresponding forms related to (2.13) read

$$
\begin{aligned}
& \sigma_{1}=G_{x}+G_{u_{0}} u_{1 t}+G_{u_{1}} u_{1 x}, \\
& \sigma_{0}=G_{t}+G_{u_{0}} u_{0 t}+G_{u_{1}} u_{0 x},
\end{aligned}
$$

Comparing $\{(2.17),(2.16)\}$ with $\{(2.9),(2.10)\}$, one can find that

$$
\begin{aligned}
X & =-G_{u_{1}}, \\
T & =-G_{u_{0}}, \\
U_{1} & =G_{x}, \\
U_{0} & =G_{t} .
\end{aligned}
$$

Thus,

$$
U=G+X u_{1}+T u_{0}=G-u_{1} G_{u_{1}}-u_{0} G_{u_{0}} .
$$

Now, the only thing left to do is to determine one of the functions $G=G\left(x, t, u_{0}, u_{1}\right), H=$ $H\left(x, t, u, u_{1}\right)$ and $J=J\left(x, t, u, u_{0}\right)$ by substituting (2.13) or (2.14) or (2.15) into (2.2). The final determining equation of $H=H\left(x, t, u, u_{1}\right)$ reads,

$$
H_{t}-\left(u_{1} F\right)_{u_{1}} H_{x}+u_{1}^{2}\left(F_{u} H_{u_{1}}-F_{u_{1}} H_{u}\right)=0 .
$$

The first order linear $H$-equation (2.23) can be readily solved by means of the characteristic line method. Its general solution has the form

$$
H=h(F, x+F t+A, t+B)
$$

where $h=h\left(\tau_{1}, \tau_{2}, \tau_{3}\right)$ is an arbitrary three dimensional function of $\tau_{1}=F=F\left(u, u_{1}\right)=u_{0} u_{1}^{-1}$, $\tau_{2}=x+F t+A$ and $\tau_{3}=t+B$. $A$ and $B$ in (2.24) are determined by

$$
\begin{aligned}
& u_{1}\left(A_{u} F_{u_{1}}-F_{u} A_{u_{1}}\right)+F_{u_{1}}=0, \\
& u_{1}^{2}\left(B_{u} F_{u_{1}}-F_{u} B_{u_{1}}\right)-1=0 .
\end{aligned}
$$

Introducing $b=b\left(a, u, u_{1}\right)$ by

$$
F(a, b)=F\left(u, u_{1}\right)
$$


$A$ and $B$ in (2.24) can be written as

$$
A= \begin{cases}-\int^{u} b^{-1} \mathrm{~d} a, & F_{u_{1}} \neq 0 \\ 0, & F_{u_{1}}=0\end{cases}
$$

and

$$
B= \begin{cases}\int^{u} b^{-2} F_{b}^{-1}(a, b) \mathrm{d} a, F_{u_{1}} \neq 0 \\ u_{1}^{-1} F_{u}^{-1}, & F_{u_{1}}=0\end{cases}
$$

According to the definition of the PBS, it is enough to find one arbitrary one dimensional function for a $1+1$ dimensional first order scalar equation (2.1). Thus, the existence of a three dimensional arbitrary function (2.23) with (2.24) means that the model is a hyper-integrable model.

It is known that whence a symmetry is obtained, one can use the symmetry to find exact solutions of the model via some different ways. The first one is the so-called symmetry reduction method to find group invariant solutions. The second one is the finite group method to find new solutions from known ones.

Starting from the symmetry (2.14) with (2.24), the related group invariant solutions can be obtained by solving equation system

$$
\begin{aligned}
& u_{t}=F\left(u, u_{x}\right) u_{x}, \\
& h\left(\tau_{1}, \tau_{2}, \tau_{3}\right)=0 .
\end{aligned}
$$

In principle, for any fixed $F=F\left(u, u_{x}\right)$, the equation system (2.30) can be readily solved.

To obtain PBSs, it is enough to use a symmetry with one dimensional arbitrary function instead of three dimensional one. For simplicity, we take the function $h$ in (2.24) only being a function of $\eta \equiv \tau_{1}^{-1}=F^{-1}=u_{1} u_{0}^{-1}$. In this special case, we have

$$
G=g\left(u_{1} u_{0}^{-1}\right) u_{1} \equiv g(\eta) u_{1} .
$$

Substituting (2.31) into (2.18)-(2.22), we have

$$
\begin{aligned}
X & =-g-\eta g_{\eta}, \\
T & =\eta^{2} g_{\eta}, \\
U_{1} & =0, \\
U_{0} & =0, \\
U & =0 .
\end{aligned}
$$

In other words, the vector form of the point Lie symmetry (2.31) has the form

$$
\begin{aligned}
V & =X \partial_{x}+T \partial_{t}+U \partial_{u}+U_{0} \partial_{u_{0}}+U_{1} \partial_{u_{1}} \\
& =-(\eta g)_{\eta} \partial_{x}+\eta^{2} g_{\eta} \partial_{t}+0 \partial_{u}+0 \partial_{u_{0}}+0 \partial_{u_{1}}
\end{aligned}
$$

Now, it is ready to obtain a PBS for the $1+1$ system (2.1) via the following theorem. 
Theorem 2.1. If $u=U(x, t)$ is a seed solution of (2.1) with the conditions $U_{x} U_{t} \neq 0$ and $U_{x} / U_{t} \neq$ constant, then a PBS of (2.1) has the form

$$
u^{\prime}(x, t)=U\left(x^{\prime}(x, t), t^{\prime}(x, t)\right)
$$

with $t^{\prime} \equiv t^{\prime}(x, t), x^{\prime} \equiv x^{\prime}(x, t)$ being determined by

$$
\begin{aligned}
& t^{\prime}=t-\eta^{2} g_{\eta}, \\
& x^{\prime}=x+(\eta g)_{\eta}, \\
& \left.\eta \equiv \frac{U_{x}}{U_{t}}\right|_{t \rightarrow t^{\prime}, x \rightarrow x^{\prime}} .
\end{aligned}
$$

Proof. Theorem 2.1 can be proved directly by solving the initial value problem related to the symmetry vector (2.33). Based on the Lie's first principle, the finite transformation of the symmetry is determined by the following initial value problem,

$$
\begin{aligned}
& \frac{\mathrm{d} t(\varepsilon)}{\mathrm{d} \varepsilon}=\left(\alpha^{2} g_{\alpha}\right)(\varepsilon), \quad t(0)=t, \alpha(\varepsilon)=\frac{u_{1}(\varepsilon)}{u_{0}(\varepsilon)}, g(\varepsilon)=g(\alpha(\varepsilon)), \\
& \frac{\mathrm{d} x(\varepsilon)}{\mathrm{d} \varepsilon}=-(\alpha g)_{\alpha}(\varepsilon), \quad x(0)=x, \\
& \frac{\mathrm{d} u(\varepsilon)}{\mathrm{d} \varepsilon}=0, \quad u(0)=U, \\
& \frac{\mathrm{d} u_{1}(\varepsilon)}{\mathrm{d} \varepsilon}=0, \quad u_{1}(0)=U_{x}, \\
& \frac{\mathrm{d} u_{0}(\varepsilon)}{\mathrm{d} \varepsilon}=0, \quad u_{0}(0)=U_{t} .
\end{aligned}
$$

It is trivial that the solution of (2.36c), (2.36d) and (2.36e) possesses the form

$$
\begin{aligned}
& u(\varepsilon)=U, \\
& u_{1}(\varepsilon)=U_{x}, \\
& u_{0}(\varepsilon)=U_{t} .
\end{aligned}
$$

The result (2.37) means that the fields $u, u_{1}$ and $u_{0}$ are $V$-group invariants and $\varepsilon$ independent. Thus $\alpha(\varepsilon)$ and $g(\varepsilon)$ are also $\varepsilon$ independent. According to this fact, the solution of (2.36a) and (2.36b) possesses the form

$$
\begin{aligned}
& t(\varepsilon)=t+\varepsilon\left(\eta^{2} g_{\eta}\right), \\
& x(\varepsilon)=x-\varepsilon(\eta g)_{\eta} .
\end{aligned}
$$

Finally, for the notation simplicity, we make the following transformations

$$
\begin{aligned}
& u(\varepsilon) \rightarrow u^{\prime}, \\
& \{x, t\} \rightarrow\left\{x^{\prime}, t^{\prime}\right\}, \\
& \{x(\varepsilon), t(\varepsilon)\} \rightarrow\{x, t\}, \\
& \varepsilon g \rightarrow g .
\end{aligned}
$$

Substituting the transformation (2.39) into (2.37a) and (2.38) leads to the proof of the theorem 2.1 . 
Remark 2.1. (i) For a given PDE (given $F$ in this paper), there may have several primary branches and secondary branches. For every given branch, one may use the symmetry theory (or other approaches) to find its PBS. (ii) For every given branch, using different seed solutions and different symmetries one may find PBSs in quite different forms. These formally different PBSs should be equivalent, however, it will be very difficult to prove the equivalence. (iii) The symmetry (2.24) can also be used to find the PBS in a different form but we do not discuss it further instead of giving a special example for fixed $F$.

Example. To end up this section, we offer a special example

$$
u_{t}^{2}-u_{x}^{2}-1+\cos (u)=2 \rho_{L}, \quad \rho_{L}=0 .
$$

The physical meaning of the model equation (2.40) is a special constant Lagrangian density (with the density $\rho_{L}$ ) evolution for the well-known sine-Gordon system. For the special model (2.40) with the zero density, it is clear that there are two primary branches with the form (2.1), i.e.,

$$
u_{t}=\delta \sqrt{u_{x}^{2}+1-\cos (u)},
$$

where $\delta=1$ and $\delta=-1$ are related to two different PBSs.

According to the general result of (2.24) for (2.1), the general Lie point symmetry reads,

$$
\begin{aligned}
\sigma & =g\left(\tau_{1}, \tau_{2}, \tau_{3}\right) u_{1} \\
& =g\left(\frac{u_{1}}{u_{0}}, x-\frac{u_{1} \operatorname{arctanh}\left(\cos \left(\frac{u}{2}\right)\right)}{\sin \left(\frac{u}{2}\right)}, t-\frac{u_{0} \operatorname{arctanh}\left(\cos \left(\frac{u}{2}\right)\right)}{\sin \left(\frac{u}{2}\right)}\right) u_{1},
\end{aligned}
$$

for the special example (2.40) with the auxiliary equations (2.7). The group invariant solutions related to (2.42) can be found by solving

$$
\begin{aligned}
& u_{t}^{2}-u_{x}^{2}+\cos (u)-1=0, \\
& g\left(\frac{u_{x}}{u_{t}}, x-\frac{u_{x} \operatorname{arctanh}\left(\cos \left(\frac{u}{2}\right)\right)}{\sin \left(\frac{u}{2}\right)}, t-\frac{u_{t} \operatorname{arctanh}\left(\cos \left(\frac{u}{2}\right)\right)}{\sin \left(\frac{u}{2}\right)}\right)=0,
\end{aligned}
$$

for arbitrary function $g$. To find the explicit solutions from (2.43) is still difficult for arbitrary $g$. Here, we only list a very special situation of (2.43),

$$
\begin{aligned}
& u_{t}^{2}-u_{x}^{2}+\cos (u)-1=0, \\
& x-\frac{u_{x} \operatorname{arctanh}\left(\cos \left(\frac{u}{2}\right)\right)}{\sin \left(\frac{u}{2}\right)}=0,
\end{aligned}
$$

to offer a special seed solution

$$
u=2 \arccos \left(\tanh \left(\frac{1}{\sqrt{2}} \sqrt{t^{2}-x^{2}}\right)\right) .
$$

Now, by using the theorem 2.1, one can readily find a PBS of (2.40),

$$
u=2 \arccos \left(\tanh \left(\frac{1}{\sqrt{2}} \sqrt{t^{\prime 2}-x^{\prime 2}}\right)\right)
$$


where $t^{\prime}$ and $x^{\prime}$ are determined by

$$
\begin{aligned}
& x^{\prime}=x+g(\eta)+\eta g_{\eta}(\eta), \quad \eta=-\frac{x^{\prime}}{t^{\prime}} \\
& t^{\prime}=t-\eta^{2} g_{\eta}(\eta)
\end{aligned}
$$

with an arbitrary function $g=g(\eta)$. Because of the arbitrariness of $g$ the solution $u$ with (2.47) is an implicit solution. To find some explicit ones, one has to suitably fix the arbitrary function $g$. For instance, if we simply fix $g=g(\eta)$ as

$$
g=-\frac{\varepsilon}{2} \eta
$$

the solution of (2.47) becomes an explicit one,

$$
\begin{aligned}
& x^{\prime}=x \frac{\tau^{2}+2 \tau(t+2 \varepsilon)+4(t-\varepsilon)^{2}}{\tau^{2}+2 \tau(t-\varepsilon)+4(t-\varepsilon)^{2}}, \\
& t^{\prime}=\frac{1}{6} \tau+\frac{1}{3}(t+2 \varepsilon)+\frac{2}{3} \frac{(t-\varepsilon)^{2}}{\tau}
\end{aligned}
$$

with

$$
\tau \equiv\left(54 x^{2} \varepsilon+8(t-\varepsilon)^{3}+6 \sqrt{3 \varepsilon} x \sqrt{27 x^{2} \varepsilon+8(t-\varepsilon)^{3}}\right)^{1 / 3} .
$$

In addition to the Lie point symmetries (2.24), the general 1+1 dimensional model (2.1) possesses much more general symmetries because of the existence of recursion operators [6]. However, here, we will not discuss the general higher order symmetries of (2.1).

\section{PBSs of arbitrary (n+1)-dimensional first order autonomous scalar fields}

Based on the idea mentioned in Section 1, finding a PBS of (1.1) is equivalent to finding a symmetry including one (one is enough for a first order PDE) $n$-dimensional arbitrary function, say,

$$
\sigma=\sigma\left(x_{0}=t, x_{1}, \ldots, x_{n}, u, G\left(\tau_{1}, \ldots, \tau_{n}\right)\right)
$$

where $G$ should be an arbitrary function of $n$ independent variables $\tau_{i}=\tau_{i}\left(t, x_{1}, \ldots, x_{n}\right)$.

A symmetry of Eq. (1.1), $\sigma$, is defined as a solution of its linearized equation

$$
F^{\prime} \sigma \equiv\left(F_{u}+\sum_{i=0}^{n} F_{u_{x_{i}}} \partial_{x_{i}}\right) \sigma \equiv F_{u} \sigma+\sum_{i=0}^{n} F_{u_{x_{i}}} \sigma_{x_{i}}=0 .
$$

As in the (1+1)-dimensional case, $u_{x_{i}} i=0,1, \ldots, n$ are the only Lie point symmetries related to the space-time translations for the arbitrary $F$ of (1.1). In order to obtain more symmetries via Lie 
point symmetry approach, we can introduce auxiliary equation systems

$$
u_{x_{i}}=u_{i}, \quad i=0,1,2, \ldots, n .
$$

Now, the general Lie point symmetries of the equation system (1.1) and (3.3) possess the forms

$$
\begin{aligned}
\sigma & =U-\sum_{j=0}^{n} X_{j} u_{x_{j}} \\
\sigma_{i} & =U_{i}-\sum_{j=0}^{n} X_{j} u_{i, x_{j}}, i=0,1, \ldots, n,
\end{aligned}
$$

where $U, U_{i}, X_{i}, i=0,1,2, \ldots, n$ are functions of $\left\{u, x_{i}, u_{i}, i=0,1,2, \ldots, n\right\}$.

As in the 1+1-dimensional case, because of the auxiliary equations (3.3), the Lie point symmetry (3.4) can be rewritten as

$$
\sigma=G\left(u, x_{i}, u_{i}, i=0,1, \ldots, n\right) \equiv g\left(u, x_{i}, u_{i}\right) u_{0} .
$$

Before doing the detailed calculations on (3.6), we would like to point out that the arbitrary function $G \equiv G\left(u, x_{i}, u_{i}, i=0,1, \ldots, n\right)$ constitutes a closed infinite dimensional Lie algebra with the commutation relation,

$$
\left[G_{1}, G_{2}\right] \equiv G_{1}^{\prime} G_{2}-\left.G_{2}^{\prime} G_{1} \equiv \frac{d}{d \varepsilon}\left[G_{1}\left(u+\varepsilon G_{2}\right)-G_{2}\left(u+\varepsilon G_{1}\right)\right]\right|_{\varepsilon=0}=G_{3},
$$

with

$$
G_{3}=G_{1 u} G_{2}-G_{2 u} G_{1}+\sum_{j=0}^{n}\left(G_{1 u_{j}} G_{2 x_{j}}-G_{2 u_{j}} G_{1 x_{j}}+G_{1 u_{j}} G_{2 u} u_{j}-G_{2 u_{j}} G_{1 u} u_{j}\right) .
$$

From the auxiliary system (3.3), we also know that $(i=0,1,2, \ldots, n)$

$$
\begin{aligned}
\sigma_{i} & =\sigma_{x_{i}} \\
& =g_{x_{i}} u_{0}+g_{u} u_{i} u_{0}+\sum_{j=0}^{n} g_{u_{j}} u_{j, x_{i}} u_{0}+g u_{0, x_{i}} \\
& =\left(g_{x_{i}}+u_{i} g_{u}\right) u_{0}+\left(u_{0} g\right)_{u_{0}} u_{i, x_{0}}+\sum_{j=1}^{n}\left(u_{0} g_{u_{j}}\right) u_{i, x_{j}} .
\end{aligned}
$$

Comparing (3.9) with (3.5), we know that

$$
\begin{aligned}
U_{i} & =\left(g_{x_{i}}+u_{i} g_{u}\right) u_{0} \\
X_{0} & =-\left(u_{0} g\right)_{u_{0}} \\
X_{i} & =-u_{0} g_{u_{i}} .
\end{aligned}
$$

Substituting the general Lie point symmetry expression (3.6) into the symmetry definition equation (3.2), we have

$$
u_{0} g_{u}+\sum_{i=0}^{n} F_{u_{i}}\left[\left(g_{x_{i}}+u_{i} g_{u}\right) u_{0}+\left(u_{0} g\right)_{u_{0}} u_{i, x_{0}}+\sum_{j=1}^{n}\left(u_{0} g_{u_{j}}\right) u_{i, x_{j}}\right]=0 .
$$


Differentiating the model equation (1.1) with respect to $x_{i}$, we have

$$
F_{u} u_{x_{i}}+\sum_{j=0}^{n} F_{u_{j}} u_{j, x_{i}}=0
$$

i.e.,

$$
\sum_{j=0}^{n} F_{u_{j}} u_{j, x_{i}}=-F_{u} u_{i}
$$

Because of (3.14), (3.13) becomes

$$
g_{u}-g F_{u}+\sum_{i=0}^{n}\left[F_{u_{i}}\left(g_{x_{i}}+u_{i} g_{u}\right)-F_{u} u_{i} g_{u_{i}}\right]=0 .
$$

It is clear that the $g$ equation (3.15) is only a first order $2 n+2$ dimensional linear PDE with respect to the variables $\left\{u, x_{i}, u_{i}, i=0,1, \ldots, n\right\}$ where one of them, say, $u$, can be replaced by others because of the model equation (1.1). Thus, after solving the linear $g$-equation (3.15), we get the following theorem.

Theorem 3.1. Arbitrary autonomous first order scalar PDE (1.1) possesses the following symmetry

$$
\sigma=g\left(\tau_{1}, \tau_{2}, \ldots, \tau_{n}, \varphi_{0}, \varphi_{1}, \varphi_{2}, \ldots, \varphi_{n}\right) u_{0}
$$

with $G \equiv G\left(\tau_{1}, \tau_{2}, \ldots, \tau_{n}, \varphi_{0}, \varphi_{1}, \varphi_{2}, \ldots, \varphi_{n}\right)$ being an arbitrary function of the indicated variables,

$$
\begin{aligned}
\tau_{\alpha} & \equiv \frac{u_{\alpha}}{u_{0}}, \quad \alpha=1,2, \ldots, n, \\
\varphi_{i} & \equiv x_{i}+A_{i}\left(u, u_{0}, u_{1}, \ldots, u_{n}\right),
\end{aligned}
$$

where $g$ is an arbitrary function of the indicated $2 n+1$ variables and $A_{i}=A_{i}\left(u, u_{0}, u_{1}, \ldots, u_{n}\right)$ are solutions of

$$
F_{u_{i}}+\sum_{j=0}^{n} u_{j}\left(F_{u_{j}} A_{i u}-A_{i u_{j}} F_{u}\right)=0
$$

After introducing $f \equiv f\left(u, u_{0}, u_{1}, \ldots, u_{n}, b\right)$ being a solution of

$$
F\left(f, b, b \tau_{1}, \ldots, b \tau_{n}\right)=F\left(u, u_{0}, u_{1}, \ldots, u_{n}\right),
$$

the solution of (3.18) can be written as $\left(\tau_{0} \equiv 1\right)$

$$
A_{i}=\left\{\begin{array}{lr}
\left.\int{ }^{u_{0}} \frac{F_{u_{i}}}{b F_{u}}\right|_{u=f, u_{j}=b \tau_{j}, j=0,1, \ldots, n} \mathrm{~d} b, F_{u} \neq 0 \\
\frac{u F_{u_{i}}}{\sum_{j=0}^{n} u_{j} F_{u_{j}}}, & F_{u}=0,
\end{array}\right.
$$

To find group invariant solutions of (1.1), one may solve the original model and several consistent symmetry constraints,

$$
\begin{aligned}
& F=0, \\
& g_{k}\left(\tau_{1}, \ldots, \tau_{n}, \varphi_{0}, \ldots, \varphi_{n}\right)=0, \quad k=1, \ldots, m,
\end{aligned}
$$


where $g_{k}$ are functions of the indicated variables and should be consistent such that the solution set of (3.21) is not empty. However, even for consistent system (3.21), it is still difficult to solve (3.21). We will offer a special example later for fixed $F$ and $g_{k}$.

To look for a PBS of the model (1.1), it is enough to use an $n$-dimensional arbitrary function, say, $g$ is only a function of $\tau_{i}$,

$$
\sigma=g\left(\tau_{1}, \tau_{2}, \ldots, \tau_{n}\right) u_{0}
$$

Substituting the special symmetry (3.22) into (3.4) and (3.12), we have

$$
\begin{aligned}
U & =0, \\
U_{i} & =0 \\
X_{0} & =-g+\sum_{i=1}^{n} \tau_{i} g_{\tau_{i}}, \\
X_{i} & =-g_{\tau_{i}} .
\end{aligned}
$$

In other words, the equivalent symmetry vector form for the equation system (1.1) and (3.3) possesses the form

$$
V=\left(g-\sum_{i=1}^{n} \tau_{i} g_{\tau_{i}}\right) \partial_{t}+\sum_{i=1}^{n} g_{\tau_{i}} \partial_{x_{i}}+0 \partial_{u}+\sum_{i=0}^{n} 0 \partial_{u_{i}} .
$$

Similar to the theorem 2.1 , corresponding to the symmetry vector (3.27), we can obtain its finite transformation theorem.

Theorem 3.2. If $u=W\left(x_{i}, i=0,1, \ldots, n\right)$ is a solution of (1.1), so is

$$
u^{\prime}\left(x_{i}, i=0,1, \ldots, n\right)=W\left(x_{i}^{\prime}\left(x_{j}, j=0,1, \ldots, n\right), i=0,1, \ldots, n\right)
$$

with $x_{i}^{\prime} \equiv x_{i}^{\prime}\left(x_{j}, j=0,1, \ldots, n\right)$ being determined by

$$
\begin{aligned}
x_{0}^{\prime} & =x_{0}-g+\sum_{\beta=1}^{n} \eta_{\beta} g_{\eta_{\beta}}, \quad g \equiv g\left(\eta_{1}, \eta_{2}, \ldots, \eta_{n}\right), \\
x_{\alpha}^{\prime} & =x_{\alpha}-g_{\eta_{\alpha}}, \alpha=1,2, \ldots, n, \\
\eta_{\alpha} & \left.\equiv \frac{W_{x_{\alpha}}}{W_{x_{0}}}\right|_{x_{i} \rightarrow x_{i}^{\prime}, i=0,1, \ldots, n} .
\end{aligned}
$$

Because the simplicity to prove the theorem 3.2 and the similarity as the proof of the theorem 2.1, we just omit the proof procedure for the theorem 3.2.

To understand the theorem 3.2, we study a special example,

$$
\sum_{i=0}^{n} u_{x_{i}}^{2}-F(u)=2 \rho_{H},
$$

which expresses the iso-Hamiltonian density evolution for $n+1$ dimensional scalar fields with an arbitrary potential $-\frac{1}{2} F(u)$ and the constant density $\rho_{H}$. Mathematically, $\rho_{H}$ may be absorbed in the potential function $F$ without loss of generality. Various well known scalar fields such as the 
sine-Gordon model, Higgs $\left(\phi^{4}\right)$ model, T. D. Lee quark bag $\left(\phi^{4}+\phi^{3}\right)$ model, $\phi^{6}$ model, double sine-Gordon model etc. are all special case of (3.30) related to suitable selections of $F(u)$.

In order to use the theorem 3.2, we have to find a nontrivial seed solution $W$ such that $W_{x_{i}} / W_{x_{0}}$ are all functionally independent. To find such a nontrivial seed solution we may use a special symmetry reduction solution of (3.21) by fixing the consistent symmetries $g_{k}$. For the scalar field system (3.30) and (3.3), its general Lie point symmetry (3.16) becomes

$$
\begin{aligned}
\sigma & =g\left(\tau_{1}, \ldots, \tau_{n}, \phi_{0}, \phi_{1}, \ldots, \phi_{n}\right) u_{0}, \\
\tau_{i} & =\frac{u_{i}}{u_{0}} \\
\phi_{i} & =x_{i}-u_{i} G G_{u}, \quad G=G(u) \equiv \int \frac{1}{\sqrt{F(u)}} \mathrm{d} u .
\end{aligned}
$$

For the symmetry (3.31), one can prove that it constitutes a closed algebra,

$$
\left[g_{1} u_{0}, g_{2} u_{0}\right]=g_{3} u_{0},
$$

where $g_{1}, g_{2}$ are arbitrary functions of $\tau_{i}$ and $\phi_{i}$ while $g_{3}$ is related to $g_{1}$ and $g_{2}$ by

$$
g_{3}=g_{1} g_{2, \phi_{0}}-g_{2} g_{1, \phi_{0}}+\sum_{i=1}^{n}\left\{\left(g_{1, \phi_{0}} g_{2, \tau_{i}}-g_{2, \phi_{0}} g_{1, \tau_{i}}\right) \tau_{i}+g_{1, \tau_{i}} g_{2, \phi_{i}}-g_{2, \tau_{i}} g_{1, \phi_{i}}\right\}
$$

For simplicity, we just look for a special symmetry reduction solution by taking (3.21) in the form

$$
\begin{aligned}
& \sum_{i=0}^{n} u_{x_{i}}^{2}-F(u)=0, \\
& \phi_{i}=x_{i}-u_{i} G G_{u}=0, i=0,1, \ldots, n .
\end{aligned}
$$

It is straightforward to integrate (3.34) one by one, and the final result reads

$$
u=W= \pm G^{-1}\left(\sqrt{\sum_{i=0}^{m} x_{i}^{2}}\right),
$$

where $G^{-1}$ is the inverse function of $G$. For instance, for the well-known scalar Higgs $\left(\phi^{4}\right)$ model

$$
F(u)=\frac{b}{4 a} u^{4}+a u^{2}+c, \quad c=0,
$$

(3.35) becomes an instanton (Rogue wave) solution

$$
u= \pm \frac{4 a \operatorname{sech}^{2}\left(\frac{\sqrt{a}}{2} \sqrt{\sum_{i=0}^{n} x_{i}^{2}}\right)}{(b-1) \tanh ^{2}\left(\frac{\sqrt{a}}{2} \sqrt{\sum_{i=0}^{n} x_{i}^{2}}\right)-2(b+1) \tanh \left(\frac{\sqrt{a}}{2} \sqrt{\sum_{i=0}^{n} x_{i}^{2}}\right)+b-1} .
$$

Now, it is ready to find a PBS for the scalar field model (3.30) by means of the seed solution (3.35) and the theorem 3.2. For the seed solution (3.35), we have

$$
\eta_{\alpha}=\left.\frac{W_{x_{\alpha}}}{W_{x_{0}}}\right|_{x_{i} \rightarrow x_{i}^{\prime}}=\frac{x_{\alpha}^{\prime}}{x_{0}^{\prime}}
$$


The related PBS of (3.30) possesses the form

$$
u= \pm G^{-1}\left(\sqrt{\sum_{i=0}^{m}\left(x_{i}^{\prime}\right)^{2}}\right)
$$

where $x_{i}^{\prime}=x_{i}^{\prime}\left(x_{0}, \ldots, x_{n}\right)$ are solutions of

$$
\begin{aligned}
x_{0}^{\prime} & =x_{0}-g+\sum_{\beta=1}^{n} \eta_{\beta} g_{\eta_{\beta}}, \quad g \equiv g\left(\eta_{1}, \eta_{2}, \ldots, \eta_{n}\right), \\
x_{i}^{\prime} & =x_{i}-g \eta_{i}, i=1,2, \ldots, n, \\
\eta_{i} & =\frac{x_{i}^{\prime}}{x_{0}^{\prime}} .
\end{aligned}
$$

with $g$ being an arbitrary function of $\eta_{i}, i=1, \ldots, n$. To give a further concrete example, we select the arbitrary function $g$ as

$$
g=a_{\alpha} \eta_{\alpha}^{2}+\sum_{\beta \neq \alpha} a_{\beta} \eta_{\beta}
$$

where $a_{i}, i=1, \ldots, n$ are arbitrary constants.

In this special case the solution of (3.40) reads

$$
\begin{aligned}
& x_{0}^{\prime}=-2 a_{\alpha}+\frac{1}{6 p}\left[4\left(t-2 a_{\alpha}\right)^{2}+2 p\left(t-2 a_{\alpha}\right)+p^{2}\right], \\
& x_{\alpha}^{\prime}=\left(1-\frac{12 a_{\alpha} p}{4\left(t-2 a_{\alpha}\right)^{2}+2 p\left(t-2 a_{\alpha}\right)+p^{2}}\right) x_{\alpha}, \\
& x_{\beta}^{\prime}=x_{\beta}-\alpha_{\beta}, \quad \beta \neq \alpha,
\end{aligned}
$$

where

$$
\left.p \equiv\left[8\left(t-2 a_{\alpha}\right)^{3}+108 a_{\alpha} x_{\alpha}^{2}+12 x_{\alpha} \sqrt{3 a_{\alpha}\left(27 a_{\alpha} x_{\alpha}^{2}+4\left(t-2 a_{\alpha}\right)^{3}\right.}\right)\right]^{1 / 3} .
$$

The PBS structure of (3.39) with (3.40) is quite complicated because of the existence of $n$ dimensional arbitrary function $g$. If the function $G^{-1}(r)$ possesses the "boundary" condition,

$$
G^{-1}(\infty)=\text { constant }
$$

the solution (3.39) with (3.40) (like the solution (3.37) with (3.42) for the $\phi^{4}$ field) possesses instanton (rogue wave like) structure.

\section{Summary and discussions}

In summary, Lie symmetry algebra (group) method is very useful to find solutions of scientific problems. In this paper, the PBSs of arbitrary first order autonomous scalar PDEs have been obtained by means of the Lie point symmetry approach. A special form of PBSs of arbitrary first order autonomous scalar PDEs, which include various important physically important special cases, is given in Theorem 3.2. The only model dependent information is included in their seed solutions. In fact, the symmetry of (1.1) obtained here includes a $2 n+1$ dimensional arbitrary function instead of an $n+1$ dimensional one. Thus we call the model (1.1) is hyper-integrable system. 
The general iso-Hamiltonian density systems for the scalar field models are explicitly studied. One special PBS is uniformly expressed by (3.39) with (3.40). For suitable selections of the boundary conditions of the Hamiltonian density, there must be one or more instanton (rogue wave like) solution(s) if the potential related inverse $G$ function possesses constant boundary (3.43) at $r=\sqrt{\sum_{i} x_{i}^{2}}=\infty$.

It is expected that the symmetry group method proposed here can also be extended to find PBSs of most general first order nonautonomous PDEs which could be solved by means of other methods such as the so-called complete solution and parameterization method [9] and the invariant function method [6]. It is also interesting that the symmetry method can be used to find PBSs and/or complete solutions for higher order nonlinear PDEs. We will report more results of applying the symmetry group method to find PBSs of PDEs in our future researches.

\section{Acknowledgement}

The author is grateful to thank Professors R. Conte, C. W. Cao, Y. Q. Li, Y. Chen, Q. P. Liu, X. B. Hu, D. J. Zhang, B. F. Feng, Z. J. Qiao and E. G. Fan for their helpful suggestions and fruitful discussions. The work was sponsored by the Global Change Research Program of China (No.2015CB953904), Shanghai Knowledge Service Platform for Trustworthy Internet of Things (No. ZF1213), the National Natural Science Foundations of China (Nos. 11435005, 11471004, 11175092, and 11205092) and K. C. Wong Magna Fund in Ningbo University.

\section{References}

[1] R. Conte, Invariant Painlevé analysis of partial differential equations, Phys. Lett. A 140 (1989) 383-389.

[2] B. Fuchssteiner, Application of hereditary symmetries to nonlinear evolution equations, Nonlinear Anal. Theor. Math. Appl. 3 (1979) 849-862.

[3] E. Hopf, The partial differential equation $u_{t}+u u_{x}=\mu u_{x x}$, Communs. Pure and Appl. Math. 3 (1950) 201-230.

[4] E. Kamke, Differentialgleichungen: Lösungsmethoden und Lösungen, II, Partielle Differentialgleichungen Erster Ordnung für eine gesuchte Funktion (Akad. Verlagsgesellschaft Geest \& Porfig Leipzig 1965).

[5] S. Y. Lou, Extended Painlevé expansion, nonstandard truncation and special reductions of nonlinear evolution equations, Z. Naturforsch. A $\mathbf{5 3}$ (1998) 251-258.

[6] S. Y. Lou and R. X. Yao, Primary branch solutions of first order autonomous scalar partial differential equations, Commun. Theor. Phys. (2017) in press (arXiv:1402.6938v3.math-ph. 2014).

[7] O. A. Oleinik, Discontinuous solutions of nonlinear differential equations, Uspekhi. Metem. Nauk. 12 (1957) 3-93 [Amer. Math. Soc. Translation. Ser 226 (1963) 95-172].

[8] P. J. Olver, Application of Lie Groups to Differential Equation, Graduate Texts in Mathematics, 2nd ed. (Springer-Verlag, NewYork, 1993).

[9] A. D. Polyanin, V. F. Zaitsev, and A. Moussiaux, Handbook of First Order Partial Differential Equations, Taylor \& Francis, (London and New York 2002).

[10] A. Ramani, B. Grammaticos and T. Bountis, The Painlevé property and singularity analysis of integrable and non-integrable systems, Phys. Rep. 180 (1989) 159-245.

[11] A. I. Subbotin, Minimax and Viscosity Solutions of Hamilton-Jacobi Equations, (Nauka Moscow [in Russian] 1991).

[12] V. Titov, A. B. Rabinovich, H. O. Mofjeld, R. E. Thomson and F. I. Gonzalez, The global reach of the 26 December 2004 Sumatra tsunami, Science 309 (2005) 2045-2048.

[13] J. Weiss, M. Tabor, C. Carnevale, The Painlevé property for partial differential equations. J. Math. Phys. 24 (1983) 522-526.

[14] G. W. Whitham, Linear and Nonlinear Waves (Wiley, New York 1974). 